ÁREA ABIERTA .Vol.12. $n^{\circ}$ 1. MARZO 2012

http://dx.doi.org/10.5209/rev_ARAB.2012.v31.38970

Referencia: AA31.1203.158

"ESTUDIO CUANTITATIVO DE LA TELEVISIÓN Y EL VÍDEO POR WEB (WEBCASTING O WEBTV): ANÁLISIS DE LA UNIDIRECCIONALIDAD E INTERACTIVIDAD"

AUTOR: Dr. José Borja ARJONA MARTíN. Universidad de Málaga

\title{
ESTUDIO CUANTITATIVO DE LA TELEVISIÓN Y EL VÍDEO POR WEB (WEBCASTING O WEBTV): ANÁLISIS DE LA UNIDIRECCIONALIDAD E INTERACTIVIDAD
}

\section{A Quantitative Study of Television and Video by Web (Webcasting or WebTV): Analysis of the Unidirectional and Interactivity}




\title{
Resumen
}

Este trabajo representa una análisis cuantitativo sobre la base de una muestra de doscientas iniciativas audiovisuales que utilizan la World Wide Web como plataforma de difusión.

El objetivo principal del estudio consiste en establecer en nuestra muestra sus aspectos interactivos o unidireccionales - designados como dimensiones- basándonos en la definición de una serie de indicadores que marcarán la naturaleza de los servicios y aplicaciones implementados en los casos de análisis. Estas dimensiones serán contrastadas con una última variable que determinará la línea de contenidos en nuestra muestra objeto de estudio. Los datos obtenidos precisarán las diferencias en cada una de las dimensiones respecto a la variable de contenido.

Palabras clave: Webcasting, WebTV, Interactividad, unidireccionalidad, televisión, vídeo, Internet.

\begin{abstract}
This work represents a quantitative analysis based on a sample of two hundred audiovisual initiatives that use the World Wide Web as a platform for diffusion.

The main objective of the study consists in stablishing in our sample his interactive or unidirectional aspects - designated as dimensions - basing us in the definition of a series of indicators that will mark the nature of the services and applications implemented in the cases of analysis. These dimensions will be contrasted with a last variable taht will determine the line of contents in our study sample. The data obtained will detail the differences in each of the dimensions respect to the variable "content".
\end{abstract}

Key words: Webcasting, WebTV, interactivity, unidirectionality, television, video, Internet. 


\section{Introducción}

El incremento de las modalidades de vídeo y televisión por redes IP ha motivado el desvanecimiento de las líneas definitorias entre las diferentes categorías establecidas. Una de estas modalidades, denominada "WebTV", y objeto de estudio en este trabajo, designa a todas aquellas iniciativas de televisión y vídeo distribuidas mediante protocolos HTTP para su distribución por la World Wide Web, siendo imprescindible el uso de un software navegador para acceder a sus contenidos y servicios, y cuyo principal dispositivo de uso es el ordenador personal.

De entre las investigaciones más actualizadas que se relacionan directamente con nuestro objeto de estudio, destacamos los trabajos de Simpson y Greenfield (2009) sobre las tipologías de distribución de vídeo por estructuras IP, pasando a mostrar sus conclusiones más relevantes:

Para Simpson y Greenfield los términos IPTV e Internet Video parecían suficientes para definir la panorámica de servicios audiovisuales por redes IP. Asimismo las diferencias entre ambos términos parecían claras y suficientes. Sin embargo, en un contexto de vertiginosos cambios tecnológicos, las fronteras entre ambos términos se difuminan y surgen nuevos conceptos para hacer referencia a los usos y contenidos más recientes.

Desde el año 2006 han nacido gran variedad de nuevos servicios que necesitan de nuevas categorías y términos que los definan. Para completar el ámbito de los servicios audiovisuales a través del protocolo de Internet Simpson y Greenfield proponen la creación de dos nuevas categorías situadas entre IPTV e Internet Video. A estas categorías las denominaron Internet TV e $I P V O D$. Así pues, ambos autores proponen cuatro modalidades de distribución de vídeo por redes IP: IPTV, IPVOD, Internet TV e Internet Video. El siguiente cuadro muestra las características y diferencias de estos cuatro grupos.

Figura1. Clasificación de los sistemas de distribución de vídeo IP

\begin{tabular}{|c|c|c|c|c|}
\hline Service Attributes & IPTV & IPVOD & Internet TV & Internet Video \\
\hline Network Type & Private Network & Public Network & Public Network & Public Network \\
\hline Quality of Service & Managed QoS & Unmanaged QoS & Unmanaged QoS & Unmanaged QoS \\
\hline Multipoint Method & True Multicasting & Unicasting & Replicated Unicasting & Unicasting \\
\hline Key Protocols & $\begin{array}{l}\text { True Streaming RTP } \\
\text { over UDP }\end{array}$ & $\begin{array}{l}\text { Progressive } \\
\quad \text { Download+Play }\end{array}$ & $\begin{array}{l}\text { HTTP Streaming; } \\
\text { Progressive D+P }\end{array}$ & $\begin{array}{l}\text { HTTP Streaming; } \\
\text { Progressive D+P }\end{array}$ \\
\hline Viewing Devices & STB with Television & $\begin{array}{l}\text { STB with Television or } \\
\text { PC }\end{array}$ & $\begin{array}{l}\text { PC, Mobile or Network } \\
\text { Appliance }\end{array}$ & $\begin{array}{l}\text { PC, Mobile or Network } \\
\text { Appliance }\end{array}$ \\
\hline Program Choices & $\begin{array}{l}\text { Hundreds of Channels } \\
\text { of Continuous TV }\end{array}$ & $\begin{array}{l}\text { Thousands of Discrete } \\
\text { Video Files }\end{array}$ & $\begin{array}{l}\text { Thousands of Channels of } \\
\text { Continuous TV }\end{array}$ & $\begin{array}{l}\text { Millions of Discrete Video } \\
\text { Files }\end{array}$ \\
\hline User Experience & $\begin{array}{l}\text { Similar to Broadcast or } \\
\text { Cable TV }\end{array}$ & Similar to DVR or VoD & Similar to Web Surfing & Similar to Web Surfing \\
\hline $\begin{array}{l}\text { Channel Change } \\
\text { Time }\end{array}$ & Quick: 1-2 seconds & Reasonable: 5-10 seconds & Slow: $10-20$ seconds & $\begin{array}{l}\text { Slow: } 10-20 \text { seconds } \\
\quad \text { (including search time) }\end{array}$ \\
\hline $\begin{array}{l}\text { Rewind/Fast } \\
\text { Forward }\end{array}$ & No & Yes & No & Yes \\
\hline Production Values & $\begin{array}{l}\text { Professionally } \\
\text { Produced }\end{array}$ & Professionally Produced & Professionally Produced & User Generated \\
\hline Content Types & Live or Prerecorded & Prerecorded Only & Live or Prerecorded & Prerecorded Only \\
\hline Program Library & Walled Content Garden & Walled Content Garden & $\begin{array}{c}\text { Worldwide Reach; } \\
\text { Quality Varies }\end{array}$ & Viewer Beware \\
\hline Ownership Rights & $\begin{array}{l}\text { Strong, with Digital } \\
\text { Rights Management }\end{array}$ & Strong, Often with DRM & Fairly Strong & $\begin{array}{l}\text { Weak or Nonexistent; } \\
\text { Frequent Copyright } \\
\text { Violations }\end{array}$ \\
\hline Revenue Models & Paid by Subscription & $\begin{array}{l}\text { Subscription, Fee per } \\
\text { Episode or Ads }\end{array}$ & $\begin{array}{l}\text { Often Free or with } \\
\text { Advertising }\end{array}$ & $\begin{array}{l}\text { Often Free or with } \\
\text { Advertising }\end{array}$ \\
\hline Example Providers & $\begin{array}{l}\text { Local Telcos, AT\&T } \\
\text { U-Verse }\end{array}$ & $\begin{array}{l}\text { Netflix, Hulu, CBS.com, } \\
\text { ABC.com, Cartoon } \\
\text { Network }\end{array}$ & $\begin{array}{l}\text { NASA.tv, Local TV } \\
\text { Broadcasters, Mogulus, } \\
\text { mobiTV }\end{array}$ & YouTube, FaceBook \\
\hline
\end{tabular}

Fuente: Greenfield, H., y Simpson, W. (2009). "IPTV and Internet Video: Expanding the Reach of Television Broadcasting". Burlington (Massachusetts): Elsevier. Pág. 18.

AA31.1203.158 
Según estas definiciones, el concepto WebTV, en su planteamiento actual (servicios y contenidos audiovisuales por Internet para ordenadores personales), englobaría a las categorías Internet TV e Internet Video al ser las que cumplen los criterios esenciales que definen la WebTV.

Ambas categorías utilizan el protocolo HTTP Streaming, basados en el estándar Hyper Text Transfer Protocol, pero con una diferencia clave: en lugar de esperar a que el navegador solicite sucesivamente los paquetes de datos de vídeo, el servidor continúa transmitiendo la información (modo push) al navegador haya o no enviado la solicitud de recepción. Con este mecanismo se asegura una velocidad de envío de paquetes determinada por el contenido de vídeo y/o audio, no por el navegador del usuario. Con ello se proporcionan velocidades de transferencia más adecuadas para el contenido.

Internet TV e Internet Video están diseñados para su acceso principalmente por un ordenador personal, aunque algunos proveedores desarrollan sistemas para dispositivos móviles. Con mayor frecuencia, empresas fabricantes y de desarrollo, lanzan al mercado aplicaciones especializadas que pueden desviar streams (flujos de datos), recibidos en un ordenador, a una pantalla de televisión; o bien aparatos conectados directamente a Internet (véase el caso de Apple TV).

Internet TV muestra una diferencia fundamental respecto a Internet Video según Greenfield y Simpson: los usuarios acceden a canales de contenido en streaming continuo en vez de acceder a un catálogo de archivos de vídeo que puedan ser reproducidos bajo demanda. A ello hay que sumarle el tipo de producción audiovisual, donde el contenido generado por los usuarios es pieza clave en los servicios de Internet Video, mientras que la producción profesional rige la modalidad Internet TV.

\section{Objetivos}

El objetivo general de estudio consiste en determinar y analizar las dimensiones interactividad y unidireccionalidad en una muestra de doscientas WebTV, utilizando la variable "contenidos" como base para el contraste con ambas dimensiones. Éstas serán definidas por la naturaleza de los indicadores de función, es decir, el nivel de comunicación unidireccional, bidireccional o multidireccional entre usuarios y entre usuarios y administradores, lo que definiría su potencial de participación.

Sujetos a este planteamiento inicial definimos los siguientes objetivos específicos:

1. Clasificación de la muestra según la variable contenidos.

2. Definición de los indicadores de función y su agrupación según las dimensiones unidireccionalidad e interactividad.

3. Contraste entre la dimensión unidireccionalidad y la variable contenidos.

4. Contraste entre la dimensión interactividad y la variable contenidos.

Y en base a estos objetivos, se plantean las siguientes hipótesis de investigación:

1. Mediante la oferta de la WebTV se está generando la unificación de las funciones de la televisión tradicional con las de Internet.

2. Se ha modificado en gran medida la dirección típica del proceso de comunicación emisorreceptor, ampliándolo a una concepción interactiva que transforma los usos y hábitos de consumo.

3. En la Web, la comunicación audiovisual se extiende a nuevos sectores de actividad, que la utilizan como apoyo a su comunicación corporativa tanto interna como externa.

4. Asociamos en mayor medida la unidireccionalidad con medios de comunicación convencionales que emprenden proyectos para la WebTV.

5. La interactividad se desarrolla gracias a las iniciativas creadas en exclusiva para la Web, es decir, WebTV nativas. 


\section{Metodología}

Según Wimmer y Dominick (1996), una de las ventajas de la investigación cualitativa es que facilita una comprensión más profunda del fenómeno estudiado, especialmente cuando se trata un asunto novedoso. Además, explican que el investigador cualitativo es una parte integrante de los datos, es decir, que sin su participación no existirían los datos y tiene en el investigador el principal instrumento de medición. En cuanto al diseño del método hablan de un proceso que evoluciona a lo largo de todo el trabajo, es decir, el método no se cierra al inicio del estudio, sino que es susceptible de cambios y adaptaciones.

Muchos investigadores optan por combinar las ópticas cualitativa y cuantitativa, como en nuestro caso, para lograr un conocimiento más completo. Es complicado encontrar un método que encaje a la perfección con nuestro objeto de estudio y los objetivos definidos, más en nuestro caso en el que tratamos la WebTV, un fenómeno tan reciente. Se trata, conforme a lo establecido en los objetivos, de desarrollar una investigación cualitativa, utilizando técnicas propias de la metodología de observación para la clasificación y definición de la muestra de estudio; y un posterior contraste cuantitativo de referencia, para hallar similitudes y diferencias entre variables y así interpretar estas relaciones, extrayendo conclusiones que nos permitan conocer la evolución actual de la televisión y el vídeo en la Web y las posibles líneas de desarrollo en un futuro inmediato.

El presente estudio consta de dos fases bien diferenciadas, una de corte cualitativo, exploratorio; siendo la segunda un análisis estadístico y, por tanto, de carácter cuantitativo.

\section{Fase de observación}

La observación supone un proceso de inspección sistemático del objeto de estudio (Frey, Botan y Kreps, 2000) y, para ello, es necesario un trabajo previo de creación de categorías que le sirvan de guía. Según Anguera (1988) la metodología observacional requiere, pues, de un sistema categorial que constituya un subconjunto de la realidad observable del objeto de nuestro estudio y, además, una observación exploratoria para la definición de dichas categorías.

De este modo, el proceso de observación exploratoria fue utilizado en nuestro caso para familiarizarnos con el objeto de estudio, y como estrategia de identificación de las características en la selección de la muestra. Igualmente, durante esta etapa del estudio, se efectuó la definición previa de categorías e indicadores de función que posteriormente se usarían en la medición.

Una vez concretadas las características básicas de nuestro objeto de estudio, las cuales se encuentran descritas en la introducción de este artículo, se llevó a cabo la búsqueda y localización de canales WebTV. Este proceso sirvió para la selección de la muestra de estudio, en base a las características fundamentales de la WebTV. Durante esta etapa la revisión de la información publicada en Internet resultó fundamental. Asimismo, para la selección de la muestra se tuvieron en cuenta los criterios definidos por Greenfield y Simpson (2009).

Una vez concretada la muestra, compuesta por 200 WebTV, se establecieron de forma definitiva las categorías de contenido y los indicadores de función empleados en el análisis de la muestra objeto de estudio.

\section{Fase de análisis estadístico}

Esta segunda fase ha tenido como objetivo primordial la cuantificación de las categorías de contenido y los indicadores de función. En esta etapa las definiciones operacionales han de ser sencillas y nítidas, de forma que los criterios de inclusión resulten evidentes y observables a simple vista. En todo análisis observacional la pieza clave es el sistema categorial empleado para clasificar y cuantificar las unidades (Wimmer y Dominick, 1996). Para ello, se ha utilizado un sistema binario - 
asignación de valores 0 y 1 - para indicar si el caso o ítem analizado pertenece a una categoría determinada o si posee o no una propiedad o función. Es decir, asignamos valores nominales a cada uno de los indicadores y categorías de contenido, de manera que otorgamos un significado cuantitativo a los objetos con el fin de utilizar estos valores en cálculos estadísticos. Para el análisis estadístico se ha elaborado una tabla de datos utilizando el programa SPSS Statistics.

Seguidamente, pasamos a mostrar las variables de análisis empleadas (indicadores de función y categorías de contenido):

A) Indicadores de función: Determinan las características funcionales de los servicios y aplicaciones de las WebTV incluidas en la muestra de estudio. Asimismo establecerán las dimensiones unidireccionales e interactivas en dicha muestra, lo que representa el objetivo fundamental de este trabajo. A continuación se muestra la relación de indicadores:

- Emisión continua: Selección, coordinación y ordenación previa, y ajena al usuario, de programas con una duración determinada.

- Descarga de vídeo: La descarga de contenido en diversos formatos como Windows Media, Quicktime, Realplayer, o bien para distintos dispositivos como IPod, PSP o teléfonos móviles.

- Canales: Programas independientes que elaboran y gestionan su propio contenido, en vivo y/o diferido, bajo el marco general de una WebTV.

- Relación de contenidos: El usuario hallará junto al reproductor una lista de vídeos relacionados con el tema principal del documento original.

- Vídeo en directo: Hacemos referencia a la transmisión en vivo tanto una señal de televisión digitalizada, como a la transmisión de vídeo desde una webcam.

1. Categorías temáticas: Clasificar sus contenidos de acuerdo con temáticas bien diferenciadas.

2. Carga de vídeo: Ofrece a los usuarios la posibilidad de incluir producciones propias de vídeo en la oferta de la WebTV.

3. Marcas activas o nube de tags: Compone un ranking, presentado a modo de célula, de palabras clave utilizadas por los usuarios como términos de búsqueda.

4. Comentarios: La posibilidad de incluir comentarios en los post (artículos) o vídeos de la plataforma.

5. Valoración: Votar sobre la experiencia del usuario con relación a los contenidos o a otros parámetros.

6. Búsqueda de contenidos: herramientas de búsqueda capaces de localizar documentos afines a los términos usados en los descriptores.

7. Ranking de valoración y votación: A partir de los votos y las valoraciones de los usuarios, ciertas herramientas elaboran listas con los contenidos más votados y más valorados por los internautas.

8. Compartir contenidos en redes sociales y bookmarks: Publicar y realizar referencias de una determinada información (escrita o audiovisual) en servicios de agrupación o redes sociales.

9. Suscripciones RSS: Los formatos RSS permiten redifundir los contenidos mediante los sistemas definidos como agregadores.

10. Vínculos o enlaces relacionados: Redirigir mediante un enlace activo desde una web a otra relacionada o de interés para la primera.

11. Grupos: Habilitar la posibilidad de crear grupos para la asociación de usuarios bajo una temática común.

12. Foros: Todos aquellos espacios webs dedicados al diálogo y al debate asíncronos en torno a una temática común.

13. Etiquetas (tags): Los vídeos pueden ser etiquetados mediante palabras clave relacionadas con el tema central y secundarios. 
14. Insertar (embed): Integrar un vídeo en una web determinada desde una fuente ajena a la ubicación de destino.

15. Opciones para el control de la reproducción: Facilitar posibilidades de pausa, avance y retroceso del vídeo.

Seguidamente mostramos la relación de indicadores de función que componen cada una de las dimensiones:

- Unidireccionalidad: Emisión continua, descarga de vídeo, canales, relación de contenidos, vídeo en directo y categorías temáticas.

- Interactividad: Carga de vídeo, marcas activas o nube de tags, comentarios, valoración, búsqueda de contenidos, rankings de valoración y votación, compartir contenidos en redes sociales y bookmarks, suscripciones RSS, vínculos o enlaces relacionados, grupos, foros, etiquetas (tags), insertar (embed) y opciones para el control de la reproducción.

B) Contenidos: Define las líneas y objetivos programáticos de las WebTV recogidas en nuestra muestra. Esta variable o categoría se subdivide en nueve grupos:

- Institucionales: WebTV centrada en la actividad de organismos públicos o privados como herramientas de información y/o comunicación interna o externa.

- Empresariales: Representan a sociedades o compañías privadas.

- Eventos: WebTV creadas en exclusiva para la cobertura de un evento o celebración determinada.

- Sociales: Aquellos servicios que se nutren del contenido proporcionado por los propios usuarios y permiten la interacción entre éstos en el medio.

- Informativas: Dedicadas íntegramente a la información, bien especializada o genérica, en relación con la actualidad.

- Generalistas: Suponen una prolongación en Internet de las televisiones convencionales de carácter nacional o autonómico. El diseño de programación procura alcanzar a toda la masa de población.

- Locales: Al igual que la anterior subcategoría, supone la representación en Internet de las televisiones locales tradicionales.

- Variados: Proporcionan contenido múltiple con clara vocación de entretenimiento.

- Plataformas: Pone al servicio del espectador una oferta amplia y diversa de canales de televisión y/o vídeo dentro de una misma ubicación. No elaboran contenido propio, su función es de directorio de otras ofertas audiovisuales.

El propósito final del análisis estadístico reside en la cuantificación de las subcategorías de contenidos y el establecimiento de posibles relaciones significativas con las dimensiones. Para obtener los resultados en ambas dimensiones se procedió a la suma de la frecuencia de los indicadores de función en cada caso de estudio (en cada WebTV), de modo que la adición de las frecuencias nos ofrece un valor cuantitativo que permitirá analizar las diferencias entre los distintos casos de estudio. Para la variable contenidos se calcularon las puntuaciones media en cada una de las dimensiones, ofreciendo el nivel de inclusión de indicadores considerados como unidireccionales o interactivos para cada una de las subcategorías de contenidos. Las dimensiones no son excluyentes, es decir, una WebTV obtiene puntuación tanto en unidireccionalidad como en interactividad, ya que pueden presentar funciones recogidas en ambas dimensiones. Por tanto, estas dos dimensiones no pueden ser tomadas como polos o extremos de un continuo, sino como características independientes que podrán tener una mayor o menor presencia.

AA31.1203.158 


\section{Resultados}

Siguiendo lo objetivos específicos planteados al inicio de este trabajo, mostramos a continuación los resultados extraídos:

\section{Clasificación y cuantificación de la muestra según la variable contenidos:}

Como puede apreciarse en la siguiente tabla, destacan las WebTV categorizadas como "Informativas" con un $31,5 \%$, seguidas por la subcategoría "Sociales" con un $22 \%$. Ambas suman más de la mitad de los casos analizados, concretamente 107 casos $(53,5 \%)$ de 200 en total.

Tabla 1. Frecuencias y porcentajes para las subcategorías de contenidos.

\begin{tabular}{|c|c|c|c|}
\hline \multicolumn{2}{|c|}{ Contenidos } & $\begin{array}{l}\text { Frecuenci } \\
\text { a }\end{array}$ & $\begin{array}{l}\text { Porcentaj } \\
\text { e }\end{array}$ \\
\hline Válidos & $\begin{array}{l}\text { Institucional } \\
\text { Empresarial } \\
\text { Eventos } \\
\text { Social } \\
\text { Informativa } \\
\text { s } \\
\text { Generalista } \\
\text { s } \\
\text { Local } \\
\text { Variados } \\
\text { Plataformas } \\
\text { Total }\end{array}$ & $\begin{array}{l}25 \\
12 \\
5 \\
44 \\
63 \\
14 \\
9 \\
19 \\
9 \\
200\end{array}$ & $\begin{array}{l}12,5 \\
6,0 \\
2,5 \\
22,0 \\
31,5 \\
7,0 \\
4,5 \\
9,5 \\
4,5 \\
100,0\end{array}$ \\
\hline
\end{tabular}

Fuente: Elaboración propia.

Los datos revelan el surgimiento de un sector emergente conformado por las iniciativas "Institucionales" (12,5\%), "Empresariales" (6\%) y "Eventos" (2,5\%). Sumados sus porcentajes se observa que ocupan el $21 \%$ del total, es decir, 42 casos.

Destacados por su origen mayoritariamente privado: compañías que invierten en el desarrollo de proyectos dirigidos generalmente al entretenimiento, las subcategorías "Plataformas" y "Variados" suman el $14 \%$ de los casos recogidos en la muestra (4,5\% y $9,5 \%$ respectivamente).

Por último, las subcategorías "Generalistas" y "Locales" obtienen un $7 \%$ (14 casos) y un 4,5\% (9 casos) respectivamente, lo que se traduce en 23 casos sobre 200 en la suma de ambas subcategorías.

\section{Cuantificación y cálculo de medias para los indicadores de función}

La siguiente tabla recoge los datos obtenidos en cada uno de los indicadores. Las funciones "opciones de reproducción" y "categorías temáticas" son las más implementadas con una media de 0,86 y 0,73 respectivamente. Por el contrario, "foros" y "grupos" son los indicadores menos representativos en el total de los 200 ítems estudiados. 
Tabla 2. Frecuencias y media según indicadores de función.

\begin{tabular}{|l|l|l|l|}
\hline & N & Suma & Media \\
\hline Opciones reproducción & 200 & 172 &, 86 \\
Categorías temáticas & 200 & 145 &, 73 \\
Búsqueda contenidos & 200 & 127 &, 64 \\
Emisión continua & 200 & 114 &, 57 \\
RSS & 200 & 75 &, 38 \\
Video en directo & 200 & 71 &, 36 \\
Vínculos & 200 & 65 &, 33 \\
Comentarios & 200 & 61 &, 31 \\
Insertar (Embed) & 200 & 61 &, 31 \\
Compartir contenidos & 200 & 60 &, 30 \\
Carga de vídeo & 200 & 56 &, 28 \\
Relación contenidos & 200 & 53 &, 27 \\
Valoración & 200 & 51 &, 26 \\
Etiquetas (Tags) & 200 & 51 &, 26 \\
Rankings valoración y & & 48 &, 24 \\
votación & 200 & 42 &, 21 \\
Canales & 200 & 42 &, 15 \\
Descarga & 200 & 30 &, 13 \\
Marcas activas & 200 & 25 &, 11 \\
Foros & 200 & 21 &, 07 \\
Grupos & 200 & 13 & \\
N válido (según lista) & 200 & & \\
Fuente Elaboración propia & & &
\end{tabular}

\section{Contraste entre la dimensión unidireccionalidad y la variable contenidos}

En el contraste entre la variable contenido y la dimensión unidireccionalidad observamos que la subcategoría "Plataformas" obtiene la mayor puntuación (3,3333; recordemos en una escala de 0 a 6), siendo "Informativas" la subcategoría con menor valor medio en unidireccionalidad $(1,8889)$. La diferencia de puntuación entre ambas subcategorías es de 1,4444.

Tabla 3. Valores medios en unidireccionalidad.

ANOVA F=3,887; Sig. $=.000$

\begin{tabular}{|l|l|l|}
\hline & $\mathrm{N}$ & Media \\
\hline Institucional & 25 & 2,2400 \\
Empresarial & 12 & 2,0000 \\
Eventos & 5 & 2,2000 \\
Social & 44 & 2,5000 \\
Informativas & 63 & 1,8889 \\
Generalista & 14 & 2,4286 \\
Local & 9 & 2,5556 \\
Variados & 19 & 2,5263 \\
Plataformas & 9 & 3,3333 \\
Total & 200 & 2,2750 \\
\hline
\end{tabular}

Fuente: Elaboración propia. 
Pese a situarse los valores medios próximos entre sí, aparentemente, las diferencias entre las subcategorías de contenidos resultan estadísticamente significativas (ANOVA F=3,887; Sig.=.000). La segunda subcategoría con mayor media es "Locales" con un 2,5556; muy próxima a "Variados" cuyo resultado medio es 2,5263, diferenciándose en tan sólo 0,0293 puntos. Por último, entre las subcategorías con mayor media en unidireccionalidad, podríamos incluir a "Sociales" con un valor medio de 2,5 indicadores de función para los 44 casos clasificados bajo este subgrupo.

Por debajo del 2,5 de media se sitúan las subcategorías "Generalistas" $(2,4286)$, "Institucionales" $(2,2400)$, "Eventos" $(2,2000)$, "Empresariales" $(2,0000)$ y la anteriormente mencionada "Informativas" $(1,8889)$.

\section{Contraste entre la dimensión interactividad y la variable contenidos}

Para la dimensión interactividad la subcategoría "Sociales" destaca de forma notable sobre el resto con una media de 9,7727 (en una escala de 0 a 14), mientras que la subcategoría "Locales" destaca en el sentido inverso con una media del 0,8889. La media total en la variable contenidos es del 4,4300.

Tabla 4. Valores medios en interactividad.

\begin{tabular}{|c|c|c|}
\hline & $\mathrm{N}$ & Media \\
\hline Instituciona & 25 & 2,2400 \\
\hline Empresarial & 12 & 3,1667 \\
\hline Eventos & 5 & 2,8000 \\
\hline Social & 44 & 9,7727 \\
\hline $\begin{array}{l}\text { Informativa } \\
\mathrm{s}\end{array}$ & 63 & 2,9048 \\
\hline $\begin{array}{l}\text { Generalist } \\
a\end{array}$ & 14 & 2,3571 \\
\hline Local & 9 & 8889 \\
\hline Variados & 19 & 5,2105 \\
\hline $\begin{array}{l}\text { Plataforma } \\
s\end{array}$ & 9 & 2,7778 \\
\hline Total & 200 & 4,4300 \\
\hline
\end{tabular}

ANOVA F=35,843; Sig. $=.000$

Como segunda subcategoría con mayor media en la inclusión de indicadores interactivos se encuentra "Variados" con un 5,2105, obteniendo 4,5622 puntos menos que "Sociales". Realzando aún más si cabe la preponderancia de esta última subcategoría en la dimensión interactiva.

Las subcategorías restantes se mantienen entre los 3 y 2 puntos de media, destacando "Empresariales" con un 3,1667 de media e "Institucionales" con un 2,2400 como subcategoría de menor representación después de "Locales". Todas estas relaciones y diferencias se confirman estadísticamente (ANOVA F=35,843; Sig.=.000). 


\section{Conclusiones}

Los datos arrojados por este estudio confirman a los medios de corte informativo como la subcategoría de contenidos más representativa de nuestra muestra, alcanzando una diferencia significativa respecto a la segunda subcategoría de mayor presencia, "Sociales". Esta última representa el relevante aspecto "social" que caracteriza a la Web desde el auge de las aplicaciones y redes sociales, traducido en un $22 \%$ ( 44 casos) de nuestra muestra.

Las subcategorías de contenido "Institucionales", "Empresariales" y "Eventos" confirman la extensión de la producción audiovisual a sectores hasta ahora ajenos a estas formas de comunicación. La suma de estas tres subcategorías constituyen un significativo $21 \%$ del total de casos estudiados, como se describió en el apartado dedicado a los resultados.

Respecto a las funcionalidades, se demuestra cierta compatibilidad entre factores que contribuyen a la participación activa de los usuarios en el medio, con aquellas funciones típicas de los medios convencionales. Algunas de las iniciativas de nuestra muestra combinan una emisión continua programada, al estilo convencional, con herramientas de búsqueda de archivos de vídeo. Recordemos que el indicador de función "emisión continua" obtiene una media de inclusión del 0,57 , y "búsqueda de contenidos" asciende a una media del 0,64.

Según los datos alcanzados, la unidireccionalidad se asocia en mayor medida a medios tradicionales que utilizan Internet como canal de difusión secundario: las subcategorías "Generalistas" y "Locales" representan por sí mismas la extensión en la Web de canales convencionales de televisión; y "Plataformas" reúne en un mismo espacio una amplia oferta de estos canales televisivos. Sin embargo, al no ser excluyentes las dimensiones, observamos como "Variados" y "Sociales" obtienen mayor media que "Generalistas" en unidireccionalidad. Esto se debe a que muchos de los casos clasificados como "Sociales" incluyen la opción de generar canales de emisión continua a sus usuarios, permitiendo personalizar la clásica forma de emisión programada de la televisión.

En relación a la dimensión interactiva de nuestra muestra, sobresale, como mencionábamos previamente, la subcategoría "Sociales". Motor de desarrollo de las opciones de participación en la WebTV; un espacio donde fomentar la comunicación audiovisual destinada a un colectivo o, por el contrario, un medio dedicado a comunicaciones interpersonales. La prometida televisión interactiva se está gestando en nuestros ordenadores personales y a través de la Web, por el impulso de las iniciativas generadas únicamente para su difusión por Internet (WebTV nativa).

Esta última dimensión confirma un bajo nivel de inclusión de indicadores interactivos en las subcategorías representativas de los medios convencionales para el conjunto de nuestra muestra de estudio ("Generalistas", 2,3571; "Locales", 0,8889).

Por su parte, las subcategorías "Institucionales", "Empresariales" y "Eventos" muestran cierta dualidad en su concepción como medio de comunicación para Internet. Algunos casos analizados pretenden emular a los canales de televisión convencionales, mientras que otros muestran señales de incorporación de elementos propios de la comunicación vía Web.

Las características de la dimensión unidireccional se incluyen de forma homogénea entre todas las subcategorías de contenido, si bien sus diferencias resultan estadísticamente significativas, destacando los subgrupos "Plataformas" y "Locales".

Respecto a la dimensión interactiva de nuestra muestra, ésta se presenta mucho más delimitada en aquellas subcategorías que favorecen a los conceptos de comunidad y cooperación. Sus diferencias resultan mucho más determinantes, sobretodo por los valores registrados en la subcategoría "Sociales".

Según los datos extraídos para el conjunto de la muestra de estudio, se confirma la extrapolación hacia la Web de conceptos clásicos de la televisión por parte de los medios convencionales. Su actividad en los nuevos medios se sostiene sobre la base de la incertidumbre. Por una parte, no supone su prioridad de negocio, aunque sí una posible ventana multiplicadora de dicho negocio. Por tanto, la lógica y la tradicional tendencia nos empuja a pensar en los medios digitales y nativos 
como la vanguardia de la innovación. Así es, y así lo confirman los datos alcanzados en este estudio. Es decir, se pone de manifiesto, de manera cuantitativa, una observación de las tendencias innovadoras en el ámbito de la comunicación audiovisual.

La WebTV se organiza por cooperación. Dicho de otro modo, los contenidos circulan en función a la interacción con el usuario. Indicadores de función como "buscar contenidos", "rankings de valoración", "compartir en redes sociales", "descarga de vídeo" o "insertar (embed)", proporcionan información directa sobre las preferencias de los usuarios respecto a los contenidos. Mediante los rankings de valoración, por ejemplo, otros usuarios pueden acceder a un catálogo de vídeos clasificados según el interés que suscita en otros usuarios.

Además, la difusión de los contenidos puede ser amplificado gracias a su efecto viral sobre los usuarios. De forma muy sencilla podemos insertar un vídeo de cualquier otro medio, en nuestro perfil de Facebook. De igual manera, lo podríamos incorporar a nuestra Web o blog personal. Por tanto, la WebTV no es ajena al concepto viral de la comunicación.

Pero la hibridación se confirma gracias a los datos arrojados por este estudio. Lo mencionábamos previamente, las opciones interactivas se fusionan con las unidireccionales. No olvidemos que el usuario demanda también un flujo de comunicación convencional, no sólo capacidades interactivas. El usuario quiere ver televisión, cine o vídeo de manera interactiva, sí, pero también de forma programada y dirigida. Los datos confirman esta dualidad, que alcanza un equilibrio relativamente estable sobretodo en las WebTV sociales. Por consiguiente, el concepto clave en el planteamiento de las estrategias de las empresas recae en la hibridación de las funcionalidades.

La WebTV constituye un vasto archivo o catálogo de contenidos de vídeo, en su gran mayoría relacionados entre sí mediante metadatos o etiquetas (tags). Considerando, además, que el catálogo se alimenta de producción profesional y producción amateur y personal.

$\mathrm{Se}$ establecen circuitos que conectan cientos de vídeos gracias a las palabras clave que los describen. Esta relación de contenidos no es si no la incorporación al vídeo en la Web de la tradicional hipertextualidad, donde un vídeo nos lleva a otros vídeos, y estos otros a muchos otros vídeos relacionados.

La WebTV representa, pues, la culminación de la televisión interactiva, pero no en los televisores, sino en nuestro ordenadores personales. El siguiente paso consiste en trasladarla a la pantalla de televisión, operación que se está llevando a cabo en las llamadas Smart TV, pero que a diferencia de la WebTV, no hay todavía una o más de una en cada hogar.

\section{Bibliografía}

AGUADO, J.M. y MARTINEZ, I. J. (2008) Sociedad móvil. Madrid: Biblioteca Nueva.

ANGUERA, M. T. (1988). Observación en la escuela. Barcelona: Editorial Graó.

BURNETT, R. y MARSHALL, P. D. (2003). Web theory. An introduction. London: Routledge.

CABERO, J. (2005) ¿̇Pero sigue existiendo la televisión? La televisión en un Universo global de la Comunicación. Comunicar, 25, pp. 57-62, ISSN: 1134-3478.

CEBRIÁN, C. (2002). Convergencia de la televisión e Internet. Tesis Doctoral, Universidad Complutense de Madrid, España.

CEBRIÁN, M. (2004). Modelos de televisión: generalista, temática y convergente con Internet. Barcelona: Paidós.

FAHS, C. (2007). How to do everything with YouTube. Nueva York: McGraw-Hill.

FREY, L., BOTAN, C. Y KREPS, G. (2000). Investigating Communication. Needham Heights, Massachussets: Allyn and Bacon.

GODZIC, W. (2010). Various faces of interactivity: Remarks on television. Icono 14 [online], 15, 22-36. Disponible en: http://www.iconol4.net.

JACKSON, L. (2010) Facilitating Participatory Audiences. Sociable Media and PSM. En LOWE, G.F: The

AA31.1203.158 
public in Public Service Media. Goteborg: Nordicom.

KATZ, E. (2009) Introduction: The end of Television?. En SCANNELL, P., KATZ, E. y KANNIS, P. (Ed.): The end of TV? Its Impact on the World (so far). Annals of the American Academy of Political and Social Science, 625(1). Londres: Sage

KYONG, W. H. (2006). New media, old media: a history and theory reader. New York: Routledge.

LOVINK, G., \& NIEDERER, S. (Eds.). (2008). Video vortex reader: Responses to YouTube. Amsterdam: Institute of Networks Cultures.

ISLA, O. (2006). La mediamorfosis de la televisión e Internet desde la óptica macluhaniana. Colombia: Revista Colombiana de Telecomunicaciones. Vol. 16, edición 41.

MESTRE PÉREZ, R. (2005). Retos de la televisión interactiva. Televisió i radio a l'era digital, 2 (7), 673686.

MILLÁN PAREDES, T. y RUANO LÓPEZ, S. (2005). De la televisión al negocio multimedia: Las nuevas opciones digitales, Fisec-estrategias, Año I Nol, Fisec. ISSN 1669- 4015.

O'DRISCOLL, G. (2008). Next generation IPTV services and technologies. New Jersey: John Wiley \& Sons.

OWEN, B. M. (1999). The Internet challenge to television. Cambridge: Harvard University Press.

PÉREZ DE SILVA, J. (2000). La televisión ha muerto: la nueva producción audiovisual en la era de Internet: la tercera revolución industrial. Barcelona: Gedisa.

RAMONET (ed.), I.; et al. (2002). La post-televisión. Multimedia, Internet y globalización económica. Barcelona: Icaria editorial.

SIMPSON, W., \& GREENFIELD, H. (2009). IPTV and Internet Video: Expanding the Reach of Television Broadcasting. Burlington (Massachusetts): Focal Press. doi:10.1016/B978-0-240-81245-8.00018-9.

VICTORIA, J. S., \& ARJONA, J. B. (2009). WebTV: Una propuesta de conceptualización y categorización para las televisiones en Internet. [online]. Actas Icono14, NoA2, I Congreso Internacional Sociedad Digital, ISSN 1697-8293. Disponible en: http://wwww.iconol4.net.

WIMMER, R. y DOMINICK, J. (1996). La investigación científica de los medios de comunicación. Barcelona: Bosch Comunicación.

WOLTON, D. (2000). ¿̇Internet, y después? Una Teoría Crítica Sobre los Nuevos "Media". Barcelona: Gedisa.

\section{Anexo. Relación de casos de estudio}

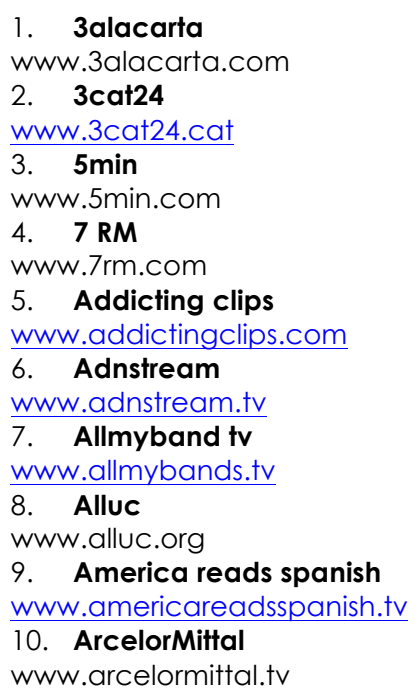




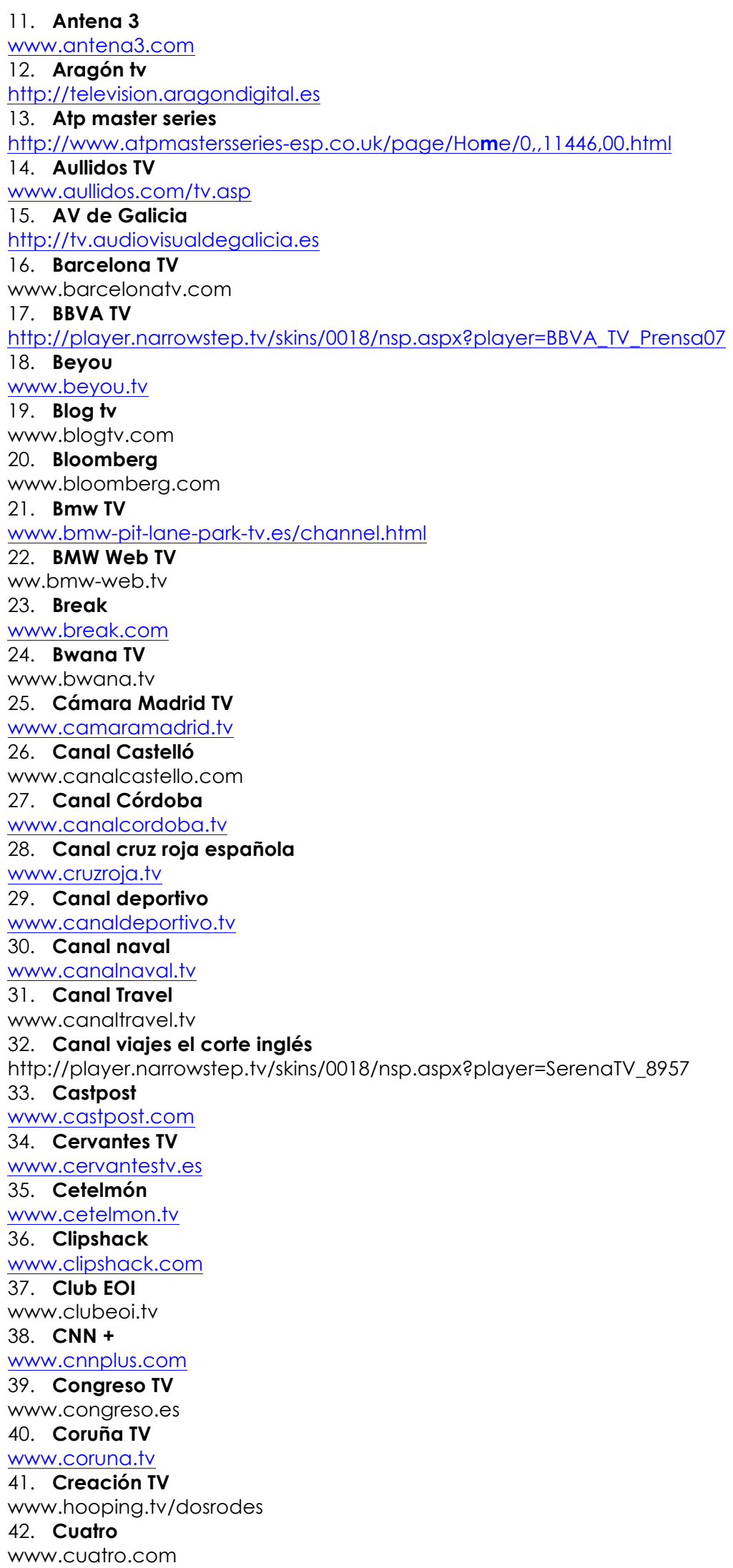


43. Culleredo TV

www.culleredo.tv

44. Cunnyfunts

www.cunnyfunts.com

45. Current TV

www.current.com

46. Channel chooser

www.channelchooser.com

47. Dailymotion

www.dailymotion.com/es

48. Dalealplay

www.dalealplay.com

49. Deusto TV

www.deusto.tv

50. Diari de Barcelona

www.diaridebarcelona.com

51. Digg

www.digg.com

52. Dos Rodes TV

www.torrenttv.tv

53. Ejido tv

www.ejidotv.com

54. EI Dia TV

www.emirasys.com/eldia.html

55. El Sol Festival TV

www.elsolfestival.tv

56. EOI Andalucia TV

wWw.andaluciaclub.tv

57. esMadrid

www.esmadrid.com

58. ETB sat

WWw.eitb.com

59. Euroleague TV

www.euroligue.tv

60. Eyespot

www.eyespot.com

61. Fadesa TV

www.fadesa.tv

62. Fotogramas TV

www.fotogramas.tv

63. Fuego TV

www.fuegotv.eu

64. Funnyordie

www.funnyordie.com

65. Generalitat Valencia

www.canalgv.com

66. Global itv

www.global-itv.com

67. Gofish

www.gofish.com

68. Google video

http://video.google.es/

69. Granollers TV

www.granollerstv.com

70. Grupo la información

www.grupolainformacion.tv

71. Guba

www.guba.com

72. Heavy

www.heavy.com

73. Hitn TV

www.hitn.tv/onlinetv

74. IBL News TV

www.iblnews.tv 


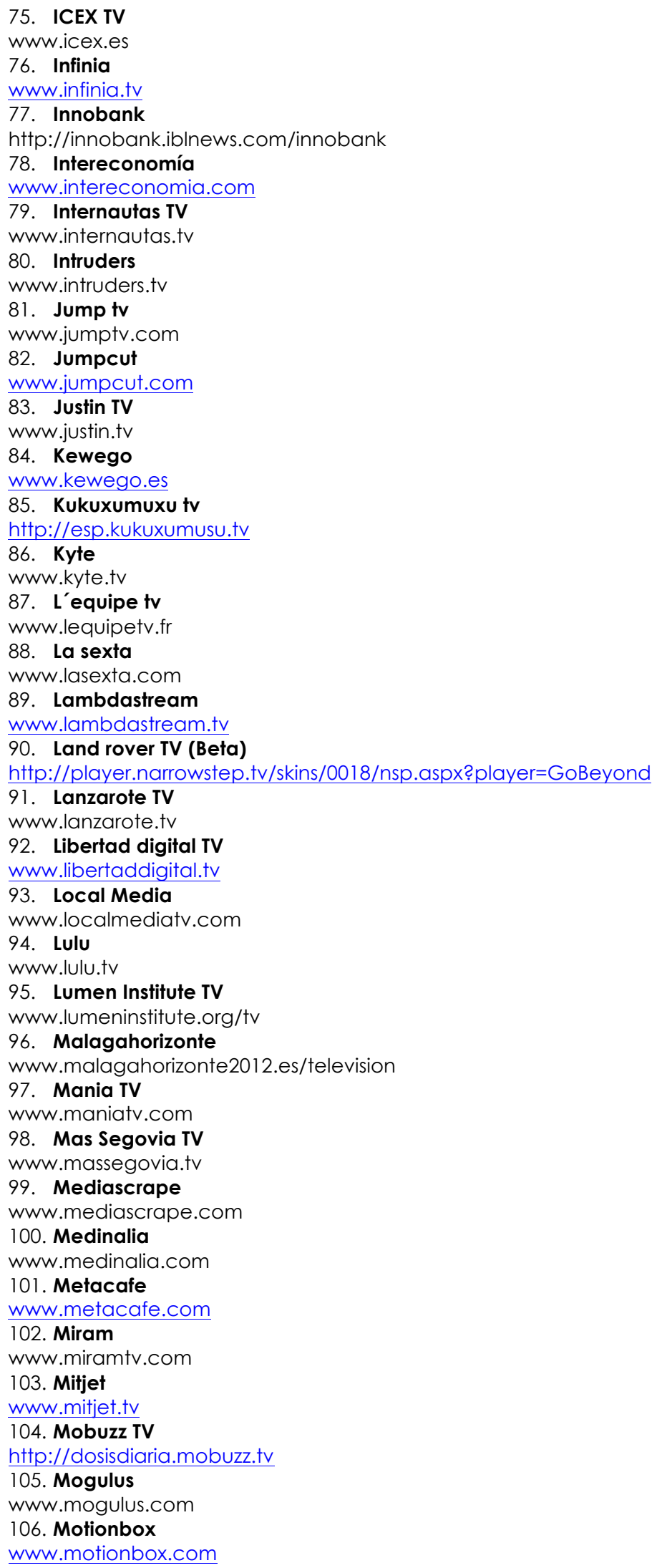


107. Motor point TV

WwW.motorpointtv.tv

108. Msn video

www.video.msn.com

109. Multiply

www.multiply.com

1 10. Mundo Franquicia TV

www.mundofranquicia.tv

111. Mundos digitales

www.mundosdigitales.tv

112. Myspace TV

www.myspacetv.com

113. Navarra TV

www.navarratv.com

114. Neave TV

www.neave.tv

115. Negopolis

www.negopolis.tv

116. Neptune

www.neptune.com

117. Nsetv

www.nsetv.com

118. Ociomadrid TV

www.ociomadrid.tv

119. Ojos solidarios

www.ojossolidarios.com

120. Onda Jerez

www.ondajerez.tv

121. Ono financiero

www.ono.com/htm/tv

122. Operator 11

www.operator1 1.com

123. Ourmedia

www.ourmedia.org

124. Pedro Halffter

www.pedrohalffter.tv

125. Peleamar tv

wWw. pleamartv.com

126. Pharmaimage tv

www.pharmaimage.tv

127. Pluràlia TV

www.pluralia.tv

128. Pobremundorico

www.pobremundorico.org

129. Pooxi

www.pooxi.com

130. Prima tv

www.prima.tv

131. Psoe tv

http://www.psoetv.es

132. Putfile

www.putfile.com

133. Quiero TV

www.quiero.tv

134. Redepyme

www.redepyme.tv

135. Reino Gourmet

www.reynogourmet.tv

136. Rocketboom

wWw.rocketboom.com

137. RTPA

Www.rtpa.es

138. RTV Insular

www.rtvinsular.com

AA31.1203.158 
139. RTVA

http://www.radiotelevisionandalucia.es/tvcarta/impe/web/portada

140. Rtve

www.rtve.es

141. RTVV

WwW.rtvv.es

142. Sagunto tv

www.sagunto.tv

143. San fermin tv

www.sanfermin.com/tv

144. Sclipo

www.sclipo.com

145. Sevenload

http://en.sevenload.com/

146. Sharkle

www.sharkle.com

147. Solidaria tv

wWw.solidariatv.com

148. Soy empresaria TV

www.soyempresaria.tv

149. Splash cast

www.splashcast.net

150. Stage6

www.stage6.com

151. Streamick

www.streamick.com

152. STV Rioja

WWW.stvrioja.com

153. Tagomedia tv

www.tagomedia.tv

154. Tb.channels TV

http://innobank.iblnews.com

155. Tele taxi

www.teletaxitv.com

156. Tele Toledo

www.teletoledo.tv

157. Telecinco

www.telecinco.es

158. Teleclip

www.teleclip.tv

159. Telemadrid

www.telemadrid.es

160. Teleweb Mallorca

www.teleweb-mallorca.com

161. Teleweb-Ibiza

www.teleweb-ibiza.tv

162. Teleweb-Menorca

www.teleweb-menorca.tv

163. Terra TV

www.terra.tv

164. Theresa Bernabé tv

www.theresabernabe.tv

165. Tinet online TV

www.tibetonline.tv

166. Tosiria RTV

www.tosiriartv.com

167. Treemo

www.treemo.com

168. Tu.tv

wWw.tu.tv

169. Tucanal tv

www.tucanaltv.com

170. Turismo Sevilla

www.turismosevilla.tv

AA31.1203.158 
171. TV Castilla y León

www.tvcyl.es

172. Tv Catalunya

http://ip.tvcatalunya.tv

173. Tv fallas

www.tvfallas.com

174. Twango

www.twango.com

175. Universidad de Coruña TV

www.udctv.es

176. Una sola tierra

www.unasolatierra.tv

177. Uncut video

http://uncutvideo.aol.com

178. UPV TV

WWW.upv.es

179. USHCC Foundation TV

www.ushccfoundationtv.org

180. Ustream

www.ustream.tv

181. Valencia Web TV

wWw.valenciawebtv.com

182. Veoh

www.veoh.com

183. Video Centre EASTV

www.videocentre.eads.net

184. Video spider

www.videospider.tv

185. Vidilife

WwW.vidilife.com

186. Vimeo

WwW.vimeo.com

187. Virgin TV

http://player26.narrowstep.tv/? player=virgin_tv

188. Visit Barcelona

www.visitbarcelona.tv

189. Vivocom.es

www.vivocom.es

190. Vmix

wWw.vmix.com

191. Vodeo

www.vodeo.tv

192. Vpod tv

http://portal.vpod.tv

193. Vsocial

www.vsocial.com

194. Wwitv

http://es.wwitv.com/

195. Yahoo video

http://es.video.yahoo.com/

196. Yendit

www.yendit.com

197. Youlike tv

www.yolike.tv

198. Youtube

http://es.youtube.com

199. Zippy videos

www.zippyvideos.com

200. Zoom in www.zoomin.tv/site/index.cfm? cid=5 